\title{
Topological Quantum Field Theories on Manifolds with a Boundary
}

\author{
Siye $\mathbf{W u} \mathbf{u}^{\star}$ \\ Department of Mathematics, Massachusetts Institute of Technology, Cambridge, \\ MA 02139, USA
}

Received March 13, 1990

\begin{abstract}
We consider a class of exactly soluble topological quantum field theories on manifolds with a boundary that are invariant on-shell under diffeomorphisms which preserve the boundary. After showing that the functional integral of the twopoint function with boundary conditions yields precisely the linking number, we use it to derive topological properties of the linking number. Considering gauge fixing, we obtain exact results of the partition function (Ray-Singer torsion of manifolds with a boundary) and the $N$-point functions in closed expressions.
\end{abstract}

\section{Introduction}

Topological field theories are highly interesting when quantized. On one hand, they provide exact solutions of physical theories like $2+1$ dimensional gravity [1]. On the other hand, they are related to a variety of topological invariants such as the Ray-Singer torsion [2,3], Donaldson invariants [4] and Jones polynomials [5]. More recently, Horowitz [6] considered a wider class of exactly soluble diffeomorphism invariant theories which include $2+1$ dimensional gravity as a special case. The action is

$$
S=\int_{M} B \wedge d C,
$$

where $B$ is a $p$-form and $C$, an $(n-p-1)$-form on an $n$ dimensional manifold $M$. Using functional integration, the two-point function was shown to be equal to the linking number of two submanifolds of $M$ of dimensions $p$ and $n-p-1[6,7]$. It was conjectured that for manifolds with a boundary, there exist boundary conditions such that the functional integral yields the same result [7].

In this letter, we consider the theory defined by action (1.1) on a manifold $M$ with boundary $\partial M$. In Sect. 2, we express the phase space as the sum of the relative

* Present address: Department of Mathematics, Columbia University, New York, NY 10027, USA 
and absolute cohomology groups of $M$ and show that the theory is invariant under diffeomorphisms which preserve the boundary, provided that the equations of motion are satisfied. We also point out the relation of Dirac quantization and Ashtekar's approach [9-11]. In Sect. 3, we incorporate the effect of boundary conditions in the functional integral. Using the Hodge decomposition on manifolds with a boundary $[12,13]$ (see also [14]), we show that the functional integral indeed yields the relative linking number of two submanifolds of $M$. In Sect. 4 , we derive several topological properties of the linking number $[16,17]$ using its path integral expression. In Sect. 5, we take into account the gauge fixing procedure. The partition function is exactly the Ray-Singer torsion with natural boundary conditions, which is a topological invariant of manifolds with a boundary $[13,15]$ (see also [3]). Furthermore, we write the $N$-point functions in closed expressions. The torsion factor cancels; this confirms the naive calculation of Sect. 3.

\section{Canonical Formalism}

For theories with gauge redundancy or symmetry, there is a covariant description of canonical formalism which manifestly preserves all relevant symmetries [18]. The classical phase space is the space of solutions of classical equations modulo the group of gauge transformations. The choice of $p s$ and $q s$ is then made according to the natural symplectic structure on the phase space.

Applying this general formalism to the theory with action (1.1), the classical equations of motions are

$$
d B=0 \text { and } \quad d C=0 ;
$$

the solutions are closed $p$-forms for $B$ and closed $(n-p-1)$-forms for $C$. The action is invariant under the following gauge transformations:

$$
B \rightarrow B+d u \text { and } C \rightarrow C+d v,
$$

where $u$ and $v$ are arbitrary $(p-1)$ - and $(n-p-1)$-forms respectively. Hence, the classical phase space is the sum of de Rham cohomology groups $[6,7]$,

$$
\mathscr{P}=H^{p}(M) \oplus H^{n-p-1}(M) .
$$

If the manifold $M$ has a boundary $\partial M$, then boundary conditions are required to kill the boundary term in the variation of the action. A $p$-form is called normal ${ }^{1}$ at the boundary if it vanishes at $\partial M$ when contracted with any tangent vectors of $\partial M$, or equivalently, it vanishes under the pullback of the inclusion map $\partial M \rightarrow M$. The space of $p$-forms satisfying the normal boundary condition is denoted by $\Omega_{\text {nor }}^{p}(M)$. A natural boundary condition of the variation of action (1.1) is that either $B$ or $C$ satisfies the normal boundary condition; we assume, without loss of generality, that $B$ does. Thus the space of classical solutions consists of closed $p$-forms $B$ with normal boundary condition and arbitrary closed $(n-p-1)$-forms $C$. Notice also that if $u \in \Omega_{\text {nor }}^{p-1}(M)$, then $d u \in \Omega_{\text {nor }}^{p}(M)$, that is, the restriction of $d$ to the forms normal at the boundary maps to those of the same type:

$$
d_{\text {nor }}: \Omega_{\text {nor }}^{p-1}(M) \rightarrow \Omega_{\text {nor }}^{p}(M) .
$$

1 The nomenclature will become clear in the next section 
The cohomology group of this complex is isomorphic to the relative cohomology group $H^{p}(M, \partial M)$, whereas the de Rham cohomology of $d$ without boundary condition is isomorphic to the absolute cohomology group $H^{p}(M)$. Modulo the gauge transformations in (2.2) with $u \in \Omega_{\mathrm{nor}}^{p-1}(M)$ and $v \in \Omega^{n-p-1}(M)$, the classical space is then

$$
\mathscr{P}=H^{p}(M, \partial M) \oplus H^{n-p-1}(M) .
$$

This space is not necessarily even dimensional even if $M$ has no boundary [6]. However, if we make the standard choice $M=\Sigma \times \mathbb{R}$ in the canonical formalism, where $\Sigma$ is an $n-1$ dimensional compact manifold with a boundary $\partial \Sigma$, then the phase space becomes

$$
\mathscr{P}=H^{p}(\Sigma, \partial \Sigma) \oplus H^{n-p-1}(\Sigma) .
$$

The even dimensionality follows from Poincaré-Lefschetz duality

$$
H^{p}(\Sigma, \partial \Sigma) \cong H^{(n-1)-p}(\Sigma) .
$$

Recall that diffeomorphisms are generated by vector fields on $M$. The infinitesimal changes of fields under a vector field $\xi$ are given by the Lie derivatives

$$
L_{\xi} B=i_{\xi} d B+d i_{\xi} B
$$

and

$$
L_{\xi} C=i_{\xi} d C+d i_{\xi} C .
$$

The first terms of the two formulas vanish if the equations of motion (2.1) are satisfied. If the infinitesimal diffeomorphism preserves the boundary, i.e., the vector field restricted to $\partial M$ is tangent to $\partial M$, then the contraction $i_{\xi}: \Omega_{\text {nor }}^{p}(M) \rightarrow \Omega_{\text {nor }}^{p-1}(M)$. Therefore, the second terms of (2.8) and (2.9) are gauge transformations generated by $u=i_{\xi} B \in \Omega_{\text {nor }}^{p-1}(M)$ and $v=i_{\xi} C \in \Omega^{n-p-2}(M)$. This shows that the theory is invariant on shell under diffeomorphisms which preserve the boundary.

Alternatively, we can take the Dirac approach: first quantize the theory, then impose the constraints. Let $t: \Sigma \times \mathbb{R} \rightarrow \mathbb{R}$ be the coordinate and $\tau$ be a vector field on $M$ such that $L_{\tau} t=1$. Then

$$
\begin{aligned}
S & =\int_{\mathbb{R}} d t \int_{\Sigma} i_{\tau}(B \wedge d C) \\
& =\int_{\mathbb{R}} d t \int_{\Sigma}(-1)^{p} B \wedge L_{\tau} C+i_{\tau} B \wedge d C+d B \wedge i_{\tau} C .
\end{aligned}
$$

So the spatial components of $B$ and $C$ are canonically conjugate variables, while the time components $i_{\tau} B$ and $i_{\tau} C$ are Lagrange multiplies enforcing the constraints

$$
C_{1}=\int_{\Sigma} u \wedge d C \text { for } u \in \Omega_{\mathrm{nor}}^{p-1}(M)
$$

and

$$
C_{2}=\int_{\Sigma} d_{\text {nor }} B \wedge v \text { for } v \in \Omega^{n-p-2}(M) .
$$

As in the case of manifolds without boundary [6], the wave functions $\psi$ are defined on the $p$-forms $B$ on $\Sigma$. The constraint $C_{2} \psi=0$ implies that $\psi$ is supported on the closed forms normal at the boundary and $C_{1} \psi=0$ implies that $\psi$ is gauge invariant. 
Define the following operators on the wave functions $\psi(B)$ :

and

$$
\mathscr{T}^{0}(\beta)=\exp \left(i \int_{\Sigma} \beta \wedge C\right)
$$

$$
\mathscr{T}^{1}(\gamma)=\int_{\Sigma} B \wedge \gamma
$$

for $\beta \in \Omega_{\text {nor }}^{p}(M), \gamma \in \Omega^{n-p-1}(M)$. Here $C$ acts as a differentiation $-i \frac{\delta}{\delta B}$, hence

$$
\mathscr{T}^{0}(\beta) \psi(B)=\psi(B+\beta) .
$$

We have a commutation relation

$$
\left[\mathscr{T}^{0}(\beta), \mathscr{T}^{1}(\gamma)\right]=-\left(\int_{\Sigma} \beta \wedge \gamma\right) \mathscr{T}^{0}(\beta)
$$

The procedure we followed is a dual version of Ashtekar's approach [9, 10], where the wave functions are complex-valued functions on homologically non-trivial loops. The loop observables satisfy a similar commutation relation $[10,11]$.

\section{The Two-Point Function with Boundary Conditions}

We now calculate the two-point function of the theory defined on a manifold $M$ with boundary $\partial M$. Since our major concern here is the role of the boundary conditions, we temporarily postpone the discussion of gauge fixing. We need to introduce a Riemannian metric on $M$. The natural inner product on $p$-forms is then

$$
\left\langle B \mid B^{\prime}\right\rangle=\int_{M} B \wedge * B^{\prime},
$$

where $*$ is the Hodge dual satisfying $* *=(-1)^{p(n-p)}$. This gives a measure of functional integration. However the final result is independent of the measure. The reason for this cancellation will become clear in the next section when we interpret the choice of the metric as fixing the gauge.

Let $U$ and $V$ be two non-intersecting submanifolds of dimensions $p$ and $n-p-1$ that represent trivial elements in $H_{p}(M, \partial M)$ and $H_{n-p-1}(M)$ respectively, that is, there exist two submanifolds $W^{\prime}$ and $W$ of dimensions $p+1$ and $n-p$ such that

$$
\left(U \backslash \partial W^{\prime}\right) \cup\left(\partial W^{\prime} \backslash U\right) \subset \partial M \quad \text { and } \quad V=\partial W .
$$

The relative linking number of $U$ and $V$ is defined to be the intersection number of $U$ and $W$

$$
L(U, V)=\sum_{p \in U \cap W} \operatorname{sign}(p),
$$

where $\operatorname{sign}(p)= \pm 1$ depending on whether or not the orientations of the tangent spaces $T_{p} U \oplus T_{p} \bar{W}$ and $T_{p} M$ agree. It follows from conditions (3.2) that $L(U, V)$ is independent of the choice of $W$. We will show that it is equal to the two-point function

$$
\left\langle\int_{U} B \int_{V} C\right\rangle=\frac{\int[\mathscr{D} B][\mathscr{D} C] \int_{U} B \int_{V} C \exp \left(i \int_{M} B \wedge d C\right)}{\int[\mathscr{D} B][\mathscr{D} C] \exp \left(i \int_{M} B \wedge d C\right)} .
$$


We need a digression on Hodge decomposition theorems on manifolds with a boundary $[12,13]$ (see also [14]). The adjoint of $d_{\text {nor }}^{p-1}$ under the inner product (3.1) is

$$
\delta^{p}=(-1)^{p n+n+1} * d^{n-p} *: \Omega^{p}(M) \rightarrow \Omega^{p-1}(M)
$$

defined on arbitrary $p$-forms (such that their images are square-integrable). However, the domain of the adjoint of $d^{p-1}$ is more restricted. A $p$-form is called tangential at the boundary if any contraction with a vector field normal to the boundary vanishes; the space of such forms is denoted by $\Omega_{\tan }^{p}(M)$. It is easy to see that

$$
*: \Omega_{\mathrm{tan}}^{p}(M) \rightarrow \Omega_{\mathrm{nor}}^{n-p}(M)
$$

and vice versa. The adjoint of $d^{p-1}$ is

$$
\delta_{\mathrm{tan}}^{p}=(-1)^{p n+n+1} * d_{\mathrm{nor}}^{n-p} *: \Omega_{\mathrm{tan}}^{p}(M) \rightarrow \Omega_{\mathrm{tan}}^{p-1}(M) .
$$

Now we have two notions of Laplacians, the relative one

$$
\Delta_{\mathrm{rel}}^{p}=d_{\mathrm{nor}}^{p-1} \delta^{p}+\delta^{p+1} d_{\mathrm{nor}}^{p}
$$

and the absolute one

$$
\Delta_{\mathrm{abs}}^{p}=d^{p-1} \delta_{\mathrm{tan}}^{p}+\delta_{\mathrm{tan}}^{p+1} d^{p} .
$$

They are elliptic self-adjoint operators defined on the space of forms with the relative boundary condition

$$
\Omega_{\mathrm{rel}}^{p}(M)=\left\{\omega \mid \omega \in \Omega_{\mathrm{nor}}^{p}(M) \text { and } \delta \omega \in \Omega_{\mathrm{nor}}^{p-1}(M)\right\}
$$

and that with the absolute boundary condition

$$
\Omega_{\mathrm{abs}}^{p}(M)=\left\{\omega \mid \omega \in \Omega_{\mathrm{tan}}^{p}(M) \text { and } d \omega \in \Omega_{\mathrm{tan}}^{p+1}(M)\right\}
$$

respectively. Hence we have two Hodge decompositions on manifolds with a boundary:

$$
\Omega^{p}(M)=d_{\text {nor }} \Omega_{\text {nor }}^{p-1}(M) \oplus \delta \Omega^{p+1}(M) \oplus \operatorname{ker} \Delta_{\text {rel }}^{p}
$$

and

$$
\Omega^{p}(M)=d \Omega^{p-1}(M) \oplus \delta_{\tan } \Omega_{\mathrm{tan}}^{p+1}(M) \oplus \operatorname{ker} \Delta_{\mathrm{abs}}^{p} .
$$

Furthermore, the space of harmonic forms with the relative (absolute) boundary condition is isomorphic to the relative (absolute) cohomology group of $M$ :

$$
\begin{aligned}
\operatorname{ker} \Delta_{\mathrm{rel}}^{p} \cong H^{p}(M, \partial M) ; \\
\operatorname{ker} \Delta_{\mathrm{abs}}^{p} \cong H^{p}(M) .
\end{aligned}
$$

We can write

$$
B=B^{L}+B^{T}+B^{0}
$$

according to the decomposition (3.12) and

$$
C=C^{L}+C^{T}+C^{0}
$$

according (3.13). Here $B^{L} \in d_{\text {nor }} \Omega_{\text {nor }}^{p-1}(M), C^{L} \in d \Omega^{p-1}(M)$ are called the longitudinal parts, $B^{T} \in \delta \Omega^{p+1}(M), C^{T} \in \delta_{\tan } \Omega_{\tan }^{p+1}(M)$ are called the transverse parts and 
$B^{0} \in \operatorname{ker} \Delta_{\mathrm{ker}}^{p}, C^{0} \in \operatorname{ker} \Delta_{\mathrm{abs}}^{p}$ are the harmonic parts of the fields [8]. Since only the transverse parts contribute to $\int_{U} B, \int_{V} C$, and $\int_{M} B \wedge d C$, the longitudinal and the harmonic parts cancel in (3.4). Hence we are led to calculate

$$
\left\langle B^{T}(x) C^{T}(y)\right\rangle=\frac{\int\left[\mathscr{D} B^{T}\right]\left[\mathscr{D} C^{T}\right] B^{T}(x) C^{T}(y) \exp \left(i \int_{M} B^{T} \wedge d C^{T}\right)}{\int\left[\mathscr{D} B^{T}\right]\left[\mathscr{D} C^{T}\right] \exp \left(i \int_{M} B^{T} \wedge d C^{T}\right)} .
$$

Let $B_{k}$ form an orthonormal basis of transverse eigenforms of the Laplacian $\Delta_{\mathrm{rel}}^{p}$ such that $\Delta_{\mathrm{rel}}^{p}=\lambda_{k}^{2} B_{k}\left(\lambda_{k}>0\right)$. The operators

$$
* d^{p}: \Omega_{\mathrm{rel}}^{p, T}(M) \rightarrow \Omega_{\mathrm{abs}}^{n-p-1, T}(M)
$$

and

$$
* d^{n-p-1}: \Omega_{\mathrm{abs}}^{n-p-1, T}(M) \rightarrow \Omega_{\mathrm{rel}}^{p, T}(M)
$$

satisfy

$$
\left(* d^{n-p-1}\right)\left(* d^{p}\right)=(-1)^{p n+1} \Delta_{\mathrm{rel}}^{p, T}
$$

and

$$
\left(* d^{p}\right)\left(* d^{n-p-1}\right)=(-1)^{p n+1} \Delta_{\mathrm{abs}}^{p, T},
$$

where the superscript $T$ means the restriction to the transverse parts. So the forms

$$
C_{k}=(-1)^{p+1} \lambda_{k}^{-1} * d B_{k} \in \Omega_{\mathrm{abs}}^{n-p-1}(M)
$$

form a basis of normalized transverse eigenforms of the Laplacian $\Delta_{\mathrm{abs}}^{n-p-1}$ with the same eigenvalues.

The rest is almost the same with the calculation in [8]. Integrating over the various modes in (3.18), we get

$$
\left\langle B^{T}(x) C^{T}(y)\right\rangle=i \sum_{k} \lambda_{k}^{-1} B_{k}(x) C_{k}(y) .
$$

Hence

$$
\begin{aligned}
\left\langle\int_{U} B \int_{V} C\right\rangle & =i \sum_{k} \lambda_{k}^{-1} \int_{U} B_{k} \int_{V} C_{k} \\
& =i \sum_{k} \int_{U} B_{k} \int_{W} * B_{k} \\
& =i L(U, V)
\end{aligned}
$$

is the linking number (times $i$ ) defined in (3.3). In the last step, we have used the completeness relation

$$
\sum_{k} B_{k}(x) \wedge * B_{k}(y)+\text { longitudinal and harmonic contributions }=\delta(x, y),(3.26)
$$

where $\delta_{y}(x)=\delta(x, y)$ is the generalized $n$-form such that for any open subset $E \subset M$,

$$
\int_{E} \delta_{y}=\left\{\begin{array}{lll}
1 & \text { if } & y \in E \\
0 & \text { if } & y \notin E .
\end{array}\right.
$$




\section{Properties of Linking Numbers via Path Integral}

We have shown

$$
L(U, V)=\frac{1}{i} \frac{\int[\mathscr{D} B][\mathscr{D} C] \int_{U} B \int_{V} C \exp \left(i \int_{M} B \wedge d C\right)}{\int[\mathscr{D} B][\mathscr{D} C] \exp \left(i \int_{M} B \wedge d C\right)} .
$$

The right-hand side is an alternative definition of the linking number which is manifestly independent of the choice of $W$ and $W^{\prime}$. This is analogous to the fact that path integral expression of the Jones polynomial is intrinsically three dimensional [5]. Though we have not considered gauge fixing, we will see in the next section that (4.1) is still correct if we have done so. We now use (4.1) to derive mathematical properties of the linking number.

First, if we reverse the orientation of $V$, using $\int_{-V} C=-\int_{V} C$, we have

$$
L(U,-V)=-L(U, V) \text {. }
$$

Consider two disjoint submanifolds $V$ and $V^{\prime}$ that do not intersect with $U$, using

$$
\int_{V \cup V^{\prime}} C=\int_{V} C+\int_{V^{\prime}} C
$$

we get

$$
L\left(U, V \cup V^{\prime}\right)=L(U, V)+L\left(U, V^{\prime}\right) .
$$

Similar properties hold for $U$.

Secondly, if we put the normal boundary condition on $C$ instead of $B$, we have

$$
L(V, U)=\frac{1}{i} \frac{\int[\mathscr{D} B]\left[\mathscr{D} C_{\text {nor }}\right] \int_{U} B \int_{V} C \exp \left(i \int_{M} B \wedge d C_{\text {nor }}\right)}{\int[\mathscr{D} B]\left[\mathscr{D} C_{\text {nor }}\right] \exp \left(i \int_{M} B \wedge d C_{\text {nor }}\right)} .
$$

Since $B \wedge d C_{\text {nor }}=(-1)^{p n+n} C_{\text {nor }} \wedge d B$ up to a total derivative, by a field redefinition $B \rightarrow(-1)^{p n+n} C, C_{\text {nor }} \rightarrow B_{\text {nor }}$ in the functional integral (4.4), we recover a well-known formula [17]

$$
L(U, V)=(-1)^{(\operatorname{dim} U+1)(\operatorname{dim} V+1)} L(V, U) .
$$

It is worth noting that if we had used another definition $L^{\prime}(U, V)$ of the linking number as the intersection number of $W^{\prime}$ and $V$, then the above formula would be [16]

$$
L^{\prime}(U, V)=(-1)^{\operatorname{dim} U \operatorname{dim} V+1} L^{\prime}(V, U) .
$$

This is also clear from the path integral point of view. If the action were replaced by $S^{\prime}=\int_{M} d B_{\text {nor }} \wedge C$, then we would use $d B_{\text {nor }} \wedge C=(-1)^{p n+1} d C \wedge B_{\text {nor }}$ to deduce (4.6).

For example, let $M=\mathbb{R}^{2}, U$ be the counterclockwise unit circle and $V=(0,0)$ $-(2,0)$ be the 0 dimensional manifold of two points with opposite orientation. Then $W^{\prime}$ is the unit disk and $W$ is the oriented segment from $(2,0)$ to $(0,0)$. It is easy to see $L(U, V)=L(V, U)=1$ while $L^{\prime}(U, V)=-L^{\prime}(V, U)=1$, which are consistent with (4.5) and (4.6). 
Finally, we show that the linking number is a cobordism invariant: if there is a submanifold $W_{1}$ such that $U \cap W_{1}=\emptyset$ and $\partial W_{1}=V \cup\left(-V^{\prime}\right)$, then

$$
L(U, V)=L\left(U, V^{\prime}\right) .
$$

In particular, the linking number is a homotopy invariant, that is, if there is a continuous deformation of submanifolds from $V$ to $V^{\prime}$ away from $U$, then (4.7) holds. The idea we use here is analogous to a hydrodynamical theorem that vorticity is conserved along vortex tubes. Consider the expectation value

$$
\langle B(x)\rangle=\frac{\int[\mathscr{D} B][\mathscr{D} C] B(x) \exp \left(i \int_{M} B \wedge d C\right)}{\int[\mathscr{D} B][\mathscr{D} C] \exp \left(i \int_{M} B \wedge d C\right)} .
$$

For any $(n-p)$-vector $\varepsilon$, the contraction $i_{\varepsilon} \delta_{y}$ is a $p$-form with normal boundary condition. After a change of variable $B \rightarrow B+i_{\varepsilon} \delta_{y}$, we get

$$
\begin{aligned}
\langle B(x)\rangle= & \frac{\int[\mathscr{D} B][\mathscr{D} C]\left[B(x)+i_{\varepsilon} \delta(x, y)\right] \exp \left[i \int_{M}\left(B+i_{\varepsilon} \delta_{y}\right) \wedge d C\right]}{\int[\mathscr{D} B][\mathscr{D} C] \exp \left(i \int_{M} B \wedge d C\right)} \\
& =\frac{\int[\mathscr{D} B][\mathscr{D} C] B(x) \exp i\left[\int_{M} B \wedge d C+i_{\varepsilon} d C(y)\right]}{\int[\mathscr{D} B][\mathscr{D} C] \exp \left(i \int_{M} B \wedge d C\right)}+i_{\varepsilon} \delta(x, y) .
\end{aligned}
$$

Differentiating with the respect to $\varepsilon$, we have

$$
0=i\langle B(x) d C(y)\rangle+\delta(x, y) .
$$

Now integrated over $x \in U, y \in W_{1}$, the delta term vanishes since $U \cap W_{1}=\emptyset$. Using Stokes' theorem, we get

$$
0=\left\langle\int_{U} B \int_{W_{1}} d C\right\rangle=\left\langle\int_{U} B \int_{V} C\right\rangle-\left\langle\int_{U} B \int_{V^{\prime}} C\right\rangle
$$

This proves (4.7). Alternatively, if we integrate over $x \in U$ and $y \in W$ where $\partial W=V$, then (4.10) yields

$$
\left\langle\int_{U} B \int_{W} d C\right\rangle=i \cdot(\text { intersection number of } U \text { and } W \text { ). }
$$

This gives a quick but naive proof of (3.25). There is no simple gauge fixing condition such that the shift $B \rightarrow B+i_{\varepsilon} \delta_{y}$ is allowed. In the next section, we will refine the derivation of Sect. 3 by considering gauge fixing.

\section{Exact Calculation of the Partition Function and $N$-Point Functions}

Quantization of theories with degenerate quadratic actions was studied a long time ago by Schwarz [2]. The general result was applied to the second order systems of antisymmetric tensor fields [3]. Though such theories are metric dependent, the ratio of two partition functions is the Ray-Singer torsion. We will 
show that the partition function of our theory with action (1.1) is indeed a topological invariant of manifolds with boundary.

We begin with a review of the Ray-Singer torsion [13,15] (see also [3]). For an $n$ dimensional Riemannian manifold $M$ with boundary $\partial M$, we define its RaySinger torsion

$$
\text { tor } M=\operatorname{det}^{\prime}\left(\delta^{n} d_{\text {nor }}^{n-1}\right)^{1 / 2} \cdot \operatorname{det}^{\prime}\left(\delta^{n-1} d_{\text {nor }}^{n-2}\right)^{-1 / 2} \cdot \operatorname{det}^{\prime}\left(\delta^{n-2} d_{\text {nor }}^{n-3}\right)^{1 / 2} \ldots,
$$

where $\operatorname{det}^{\prime}$ denotes the zeta-function regularization of the determinant with zero modes excluded. Using the factorization

$$
\operatorname{det}^{\prime}\left(\Delta_{\mathrm{rel}}^{p}\right)=\operatorname{det}^{\prime}\left(\delta^{p+1} d_{\mathrm{nor}}^{p}\right) \cdot \operatorname{det}^{\prime}\left(d_{\mathrm{nor}}^{p-1} \delta^{p}\right)
$$

we can also write

$$
\text { tor } M=\operatorname{det}^{\prime}\left(\Delta_{\mathrm{rel}}^{n-1}\right)^{1 / 2} \cdot \operatorname{det}^{\prime}\left(\Delta_{\mathrm{rel}}^{n-2}\right)^{-1} \cdot \operatorname{det}^{\prime}\left(\Delta_{\mathrm{rel}}^{n-3}\right)^{3 / 2} \ldots .
$$

Since the $*$ in (3.6) is an isomorphism, we have

$$
\operatorname{det}^{\prime}\left(\delta^{p+1} d_{\mathrm{nor}}^{p}\right)=\operatorname{det}^{\prime}\left(* \delta^{p+1} d_{\mathrm{nor}}^{p} *\right)=\operatorname{det}^{\prime}\left(d^{n-p-1} \delta_{\mathrm{tan}}^{n-p}\right)=\operatorname{det}^{\prime}\left(\delta_{\mathrm{tan}}^{n-p} d^{n-p-1}\right)
$$

Hence another form of the Ray-Singer torsion is

$$
\begin{aligned}
\operatorname{tor} M= & {\left[\operatorname{det}^{\prime}\left(\delta_{\mathrm{tan}}^{n} d^{n-1}\right)^{1 / 2} \cdot \operatorname{det}^{\prime}\left(\delta_{\mathrm{tan}}^{n-1} d^{n-2}\right)^{-1 / 2}\right.} \\
& \left.\times \operatorname{det}^{\prime}\left(\delta_{\mathrm{tan}}^{n-2} d^{n-3}\right)^{1 / 2} \ldots\right]^{(-1)^{n-1}}, \\
= & {\left[\operatorname{det}^{\prime}\left(\Delta_{\mathrm{abs}}^{n-1}\right)^{1 / 2} \cdot \operatorname{det}^{\prime}\left(\Delta_{\mathrm{abs}}^{n-2}\right)^{-1} \cdot \operatorname{det}^{\prime}\left(\Delta_{\mathrm{abs}}^{n-3}\right)^{3 / 2} \ldots\right]^{(-1)^{n-1}} . }
\end{aligned}
$$

We now calculate the partition function of the theory. We fix the gauge $B^{L}=0$ and $C^{L}=0$. Assuming that the Laplacians on $M$ have no zero modes, these gauge conditions are equivalent to

$$
\delta^{p} B=0 \quad \text { and } \quad \delta_{\tan }^{n-p-1} C=0 .
$$

The standard Faddeev-Popov determinant factors

$$
\operatorname{det}^{\prime}\left(\delta^{p} d_{\text {nor }}^{p-1}\right)^{1 / 2} \text { and } \operatorname{det}^{\prime}\left(\delta_{\text {tan }}^{n-p-1} d^{n-p-2}\right)^{1 / 2}
$$

should be modified because closed ( $p-1)$ - and $(n-p-2)$-forms generate trivial transformations. According to Schwarz's general formula $[2,3]$ on degenerate functional integration, the partition function of the action (1.1) is

$$
\begin{aligned}
Z_{p}= & \operatorname{det}^{\prime}\left(\delta^{p} d_{\mathrm{nor}}^{p-1}\right)^{1 / 2} \cdot \operatorname{det}^{\prime}\left(\delta^{p-1} d_{\mathrm{nor}}^{p-2}\right)^{-1 / 2} \cdot \operatorname{det}^{\prime}\left(\delta^{p-2} d_{\mathrm{nor}}^{p-3}\right)^{1 / 2} \ldots \\
& \times \operatorname{det}^{\prime}\left(\delta_{\mathrm{tan}}^{n-p-1} d^{n-p-2}\right)^{1 / 2} \cdot \operatorname{det}^{\prime}\left(\delta_{\mathrm{tan}}^{n-p-2} d^{n-p-3}\right)^{-1 / 2} \\
& \times \operatorname{det}^{\prime}\left(\delta_{\mathrm{tan}}^{n-p-3} d^{n-p-4}\right)^{1 / 2} \ldots \\
& \times \operatorname{det}^{\prime}\left[\left(* d^{n-p-1}\right)^{\dagger}\left(* d^{n-p-1}\right)\right]^{-1 / 2} \\
= & \operatorname{det}^{\prime}\left(\delta^{p} d_{\mathrm{nor}}^{p-1}\right)^{1 / 2} \cdot \operatorname{det}^{\prime}\left(\delta^{p-1} d_{\mathrm{nor}}^{p-2}\right)^{-1 / 2} \cdot \operatorname{det}^{\prime}\left(\delta^{p-2} d_{\mathrm{nor}}^{p-3}\right)^{1 / 2} \ldots \\
& \times \operatorname{det}^{\prime}\left(\delta^{p+2} d_{\mathrm{nor}}^{p+1}\right)^{1 / 2} \cdot \operatorname{det}^{\prime}\left(\delta^{p+3} d_{\mathrm{nor}}^{p+2}\right)^{-1 / 2} \cdot \operatorname{det}^{\prime}\left(\delta^{p+4} d_{\mathrm{nor}}^{p+3}\right)^{1 / 2} \ldots \\
& \times \operatorname{det}^{\prime}\left(\delta^{p+1} d_{\mathrm{nor}}^{p}\right)^{-1 / 2} \\
= & (\operatorname{tor} M)^{(-1)^{n-p}} .
\end{aligned}
$$

The topological invariance of the Ray-Singer torsion shows that the partition function is independent of the gauge fixing conditions. If the cohomology groups of 
the manifolds are non-trivial, then we should integrate over the harmonic forms as well. The result in (5.8) is interpreted as the partition measure on the space of zero modes of the Laplacians [3]. It follows from (5.4) that if we had put the normal boundary condition on $C$ instead of $B$, the partition function would be

$$
Z_{p}^{\prime}=\left[(\operatorname{tor} M)^{(-1)^{n-1}}\right]^{(-1)^{n-p}}=(\operatorname{tor} M)^{(-1)^{p+1}} .
$$

As a consistency check, we notice that $Z_{p}=Z_{n-p-1}^{\prime}$, since the two theories are related by an integration by parts in the action.

To calculate correlation functions of the theory, we introduce two sources $X \in \Omega_{\mathrm{rel}}^{p, T}(M)$ and $Y \in \Omega_{\mathrm{abs}}^{n-p-1, T}(M)$. Define

$$
Z_{p}[X, Y]=\int[\mathscr{D} B][\mathscr{D} C] \exp \left[i \int_{M} B \wedge d C+X \wedge * B+Y \wedge * C\right]
$$

with extra factors of determinants due to gauge fixing. Using an algebraic identity

$$
\begin{aligned}
B \wedge d C+X \wedge * B+Y \wedge * C= & \left(B+(-1)^{p n+n} d^{-1} * Y\right) \wedge d\left(C+d^{-1} * X\right) \\
& -(-1)^{p n+n} X \wedge * d^{-1} * Y,
\end{aligned}
$$

we deduce, by shifting the field variables in the functional integral, that

$$
Z_{p}[X, Y]=Z_{p} \exp \left[-i(-1)^{p n+n} \int_{M} X \wedge *\left(d_{\text {nor }}^{p, T}\right)^{-1} * Y\right]
$$

The correlation function of $r B$ fields and $s C$ fields is

$$
\begin{aligned}
\left\langle B\left(x_{1}\right) \ldots B\left(x_{r}\right) C\left(y_{1}\right) \ldots C\left(y_{s}\right)\right\rangle=Z_{p}^{-1}\left(-i \frac{\delta}{\delta X\left(x_{1}\right)}\right) \ldots\left(-i \frac{\delta}{\delta X\left(x_{r}\right)}\right)\left(-i \frac{\delta}{\delta Y\left(y_{1}\right)}\right) \\
\left.\quad \ldots\left(-i \frac{\delta}{\delta Y\left(y_{s}\right)}\right) Z_{p}[X, Y]\right|_{X=0, Y=0} \\
=\left(-i \frac{\delta}{\delta X\left(x_{1}\right)}\right) \ldots\left(-i \frac{\delta}{\delta X\left(x_{r}\right)}\right)\left(-i \frac{\delta}{\delta Y\left(y_{1}\right)}\right) \ldots\left(-i \frac{\delta}{\delta Y\left(y_{s}\right)}\right) \\
\quad \times\left.\exp \left[-i(-1)^{p n+n} \int_{M} X \wedge *\left(d_{\text {nor }}^{p, T}\right)^{-1} * Y\right]\right|_{X=0, Y=0}
\end{aligned}
$$

for $x_{1}, \ldots, x_{r}, y_{1}, \ldots, y_{s} \in M$. In particular, the two-point function is

$$
\begin{aligned}
\langle B(x) C(y)\rangle & =-\left.\frac{\delta}{\delta X(x)} \frac{\delta}{\delta Y(y)} \exp \left[-i(-1)^{p n+n} \int_{M} X \wedge * d^{-1} * Y\right]\right|_{X=0, Y=0} \\
& =i\left\langle x\left|(-1)^{p n+n}\left(d_{\text {nor }}^{p, T}\right)^{-1} *\right| y\right\rangle \\
& =i(-1)^{p n+n} \sum_{k, l}\left\langle x \mid B_{k}\right\rangle\left\langle B_{k}\left|d^{-1} *\right| C_{l}\right\rangle\left\langle C_{l} \mid y\right\rangle \\
& =i \sum_{k} \lambda_{k}^{-1} B_{k}(x) C_{k}(y) .
\end{aligned}
$$

Here, we have used (3.23) to show $\left\langle B_{k}\left|d^{-1} *\right| C_{l}\right\rangle=(-1)^{p n+n} \lambda_{k}^{-1}$ in the last step. This result is identical to formula (3.24) that was derived without considering gauge fixing. Hence our exact calculation not only yields a closed expression of the $N$-point function, but also confirms (3.25) that the two-point function is the linking number. 
Finally, if we interpret (5.14) as the propagator under gauge conditions (5.7), then (5.13) gives precisely the Feynman rule

$$
\left\langle B\left(x_{1}\right) \ldots B\left(x_{r}\right) C\left(y_{1}\right) \ldots C\left(y_{s}\right)\right\rangle=\delta_{r s} \sum_{\sigma \in S_{r}} \prod_{k=1}^{r} i\left\langle x_{k}\left|(-1)^{p n+p}\left(d_{\mathrm{nor}}^{p, T}\right)^{-1} *\right| y_{\sigma(k)}\right\rangle,
$$

where $S_{r}$ is the permutation group on $r$ letters. Integrating over submanifolds $U_{1}, \ldots, U_{r} \subset M$ of dimension $p$ and $V_{1}, \ldots, V_{s} \subset M$ of dimension $n-p-1$ such that $U_{i} \cap V_{j}=\emptyset$, we get

$$
\left\langle\int_{U_{1}} B \ldots \int_{U_{r}} B \int_{V_{1}} C \ldots \int_{V_{s}} C\right\rangle=\delta_{r s} i^{r} \sum_{\sigma \in S_{r}} \prod_{k=1}^{r} L\left(U_{k}, V_{\sigma(k)}\right) .
$$

Notice that if we had chosen the $B$ and $C$ fields to be fermionic, the above correlation function would be

$$
\delta_{r s}{ }^{r} \operatorname{det}\left[L\left(U_{i}, V_{j}\right)\right]_{i, j=1, \ldots, r} .
$$

Acknowledgements. I would like to thank D. Z. Freedman, V. Guillemin, P. Piazza, and N. Warner for encouragement and discussions. The work was supported in part by a NSF Grant PHY87-08447.

\section{References}

1. Witten, E.: $2+1$ dimensional gravity as an exactly soluble system. Nucl. Phys. B 311, 46 (1988/89); Topology-changing amplitudes in 2+1 dimensional gravity. Nucl. Phys. B 323, 113 (1989)

2. Schwarz, A.S.: The partition function of degenerate quadratic functional and Ray-Singer torsion. Lett. Math. Phys. 2, 247 (1978)

3. Schwarz, A.S., Tyupkin, Y.S.: Quantization of antisymmetric tensors and Ray-Singer torsion. Nucl. Phys. B 242, 436 (1984)

4. Witten, E.: Topological quantum field theory. Commun. Math. Phys. 117, 353 (1988)

5. Witten, E.: Quantum field theory and the Jones polynomial. Commun. Math. Phys. 121, 351 (1989)

6. Horowitz, G.T.: Exactly soluble diffeomorphism invariant theories. Commun. Math. Phys. 125, 417 (1989)

7. Blau, M., Thompson, G.: Topological gauge theories of antisymmetric tensor field. Trieste preprint SISSA-39-FM (1989)

8. Horowitz, G.T., Srednicki, M.: A quantum field theoretic description of linking numbers and their generalizations. Commun. Math. Phys. 130, 33 (1990)

9. Ashtekar, A.: New variables for classical and quantum gravity. Phys. Rev. Lett. 57, 2244 (1986); New Hamiltonian formulation of general relativity. Phys. Rev. D 36, 1587 (1987)

10. Ashtekar, A., Husain, V., Rovelli, C., Samuel, J., Smolin, L.: $2+1$ quantum gravity as a toy model for the 3+1 theory. Class. Quant. Gravity 6, 1185 (1989)

11. Husain, V.: Topological quantum mechanics. University of Utah preprint (1990), unpublished

12. Conner, P.: The Neumann's problem for differential forms on Riemannian manifolds. In: Mem. Am. Math. Soc., vol. 20. Providence, RI: Am. Math. Soc., 1956

13. Ray, D., Singer, I.: $R$-torsion and the Laplacian on Riemannian Manifolds. Adv. Math. 7, 145 (1971)

14. Wu, S.: Determinants of Dirac operators and Thirring model partition functions on Riemann surfaces with boundaries. Commun. Math. Phys. 124, 133 (1989)

15. Ray, D., Singer, I.: Analytic torsion. In: Proc. Symp. Pure Math., vol. 23, p. 167. Berkeley 1971. Spencer, D.C. (ed.). Providence, RI: Am. Math. Soc. 1979; Analytic torsion for complex manifolds. Ann. Math. 98, 154 (1973) 
16. Seifert, H., Threlfall, W.: Lehrbuch der Topologie, p. 277. New York: Chelsea 1947 Rolfsen, D.: Knots and links, p. 132. Berkeley, CA: Publish or Perish 1976

17. Milnor, J.W.: Topology from the differential point of view, p. 53. Charlottesville, VA: University Press of Virginia 1965

18. Zuckerman, G.: Action functionals and global geometry, in: Mathematical aspects of string theory, p. 259, San Diego, 1986. Yau, S.-T. (ed.). Singapore: World Scientific 1987

Crnkovic, C., Witten, E.: Covariant description of canonical formalism in geometrical theories, in: Three hundred years of gravitation, p. 676. Hawking, S.W., Israel, W. (eds.). Cambridge: Cambridge University Press 1987

Communicated by S.-T. Yau 